\title{
Quasi-invariant measures on linear topological spaces
}

\author{
By Yasuji TAKahashi
}

\section{§1. Introduction}

In [1], Dao-Xing has shown that the following:

THEOREM Let $H$ be a separable Hilbert space, and let $\mathfrak{F}$ be the totality of weak Borel sets in $H$. Let $\Phi$ be a linear subspace of $H$, and suppose that $\Phi$ itself is a complete $\sigma$-Hilbert space with respect to the sequence of inner products $(\varphi, \phi)_{n}, n=1,2,3, \cdots$

where $\quad(\varphi, \varphi)_{1} \leqq(\varphi, \varphi)_{2} \leqq \cdots$.

Also, suppose that the inclusion mapping $T$ from $\Phi$ into $H$ is continuous. For each $n$, let $\Phi_{n}$ denote the completion of $\Phi$ with respect to the inner product $(\varphi, \phi)_{n}$. Then, the following conditions are equivalent.

(1) There exists a $\Phi$-quasi-invariant finite measure (non-trivial) $\mu$ on $(H, \mathfrak{F})$.

(2) There exists $n$ such that the inclusion mapping $T$ can be extended to a Hilbert-Schmidt operator from $\Phi_{n}$ into $H$.

In the Dao-Xing's original Theorem, it is necessary that $\mu$ is regular. In this paper, we shall show that this assumption can be taken off, furthermore this theorem can be extended to complete $\sigma$-normed spaces.

Throughout this paper (except for $\S 2.1^{\circ}$. and $\S 5$.), we shall assume that linear spaces are with real coefficients.

\section{\$2. Basic definitions and well known results}

$1^{\circ}$. p-absolutely summing operators $(1 \leqq p<\infty)$

Let $E$ and $F$ be Banach spaces.

DEFINITION 2.1.1. Let $\left\{x_{n}\right\}$ be a sequence from a Banach space $E$. $\left\{x_{n}\right\}$ is called scalarly $l_{p}$ if for each continuous linear functional $x^{*} \in E^{*}$, we have the inequality

$$
\sum_{n=1}^{\infty}\left|x^{*}\left(x_{n}\right)\right|^{p}<\infty
$$

$\left\{x_{n}\right\}$ is called absolutely $l_{p}$ if $\sum_{n=1}^{\infty}\left\|x_{n}\right\|^{p}<\infty$.

Definition 2.1.2. A linear operator $T$ from $E$ into $F$ is called $p$ - 
absolutely summing if for each $\left\{x_{n}\right\} \subset E$ which is scalarly $l_{p},\left\{T\left(x_{n}\right)\right\} \subset F$ is absolutely $l_{p}$.

We shall say "absolutely summing" instead of " 1 -absolutely summing". Proposition 2.1.1. (c.f. [2])

$A$ linear operator $T$ from $E$ into $F$ is p-absolutely summing iff there is a constant $C$ such that for every finite set in $E x_{1}, x_{2}, \cdots, x_{n}$, the following

holds.

$$
\sum_{k=1}^{n}\left\|T\left(x_{k}\right)\right\|^{p} \leqq C \sup _{\left\|x^{*}\right\| \leqq 1}\left(\sum_{k=1}^{n}\left|x^{*}\left(x_{k}\right)\right|^{p}\right)
$$

Proposition 2. 1.2. (c.f. [3])

Let a linear operator $T$ from $E$ into $F$ be p-absolutely summing. If $1 \leqq p \leqq q<\infty$, then $T$ is $q$-absolutely summing.

Proposition 2.1.3. (c.f. [3])

A linear operator $T$ from $E$ into $F$ is p-absolutely summing iff there is a probability measure $\mu$ on the compact set $K^{*}=$ the $w^{*}$-closure of the set of all extreme points of the unit ball of $E^{*}$, and a constant $C$ such that

$$
\|T(x)\| \leqq C\left(\int_{K^{*}}\left|x^{*}(x)\right|^{p} d \mu\left(x^{*}\right)\right)^{\frac{1}{p}}, \text { for } x \in E .
$$

Corollary 2.1.1. (c.f. [3])

Let $T$ be a 2-absolutely summing operator. Then there is a probability measure $\mu$ on $K^{*}$ and an operator $S: L_{2}(\mu) \rightarrow F$ such that

(a) $S$ is a continuous linear operator.

(b) $T=S \circ J \circ I$, where $I: E \rightarrow C\left(K^{*}\right)$ is the canonical isometry $x \rightarrow x\left(x^{*}\right)$ and $J: C\left(K^{*}\right) \rightarrow L_{2}(\mu)$ is the identity operator.

PROPOSITION 2.1.4. (c.f. [3])

Let $K$ be a compact Hausdorff space and $\mu$ be a probability measure on $K$. Then the identity operator $I: \quad C(K) \rightarrow L_{p}(\mu)$ is p-absolutely summing.

Proposition 2.1.5. (c.f. [3], [4])

Let $H_{1}$ and $H_{2}$ be Hilbert spaces and let $T$ be a linear operator from $H_{1}$ into $H_{2}$. Then the followings are equvalent.

(a) $T$ is p-absolutely summing.

(b) $T$ is a Hilbert-Schmidt operator.

By Corollary 2.1.1. Proposition 2.1.4. and Proposition 2.1.5, we have the following Proposition.

PROPOSITION 2.1.6. (c.f. [3]) 
Let $H$ be a Hilbert space and $E$ be a Banach space. Then the followings are equivalent.

(a) $T$ is 2-absolutely summing.

(b) There exists a Hilbert space $H_{1}$ such that

$$
H \underset{U}{\longrightarrow} H_{1} \underset{V}{\longrightarrow} E
$$

$T=V \circ U$ where $U$ is a Hilbert-Schmidt operator and $V$ is a continuous linear operator.

EXAMPLE 1 . identity operator $I: l_{1} \rightarrow l_{2}$ is absolutely summing.

EXAMPLE 2. identity operator $I: \quad l_{2} \rightarrow l_{\infty}$ is not $p$-absolutely summing, for $1 \leqq p<\infty$.

REMARK. Generally, $p$-absolutely summing operator is not necessarily compact. (c.f. Ex. 1.)

But a $p$-absolutely summing operator $T$ from a Hilbert space $H$ into a Banach space $E$ is compact.

\section{$2^{\circ}$. Cylinder sets and Cylinder measures}

In this subsection, we describe certain $\sigma$-algebras which will often be used in the ensuing discussion, and examine the relations between them.

Definition 2.2.1. Let $E$ be a real linear topological space and $E^{*}$ be $a$ adjoint space of $E$. If $A$ is a Borel set in real $n$-dimensional space $R_{n}$, and $x_{1}, x_{2}, \cdots, x_{n} \in E$, the set

$$
\left\{x^{*} \mid\left(x^{*}\left(x_{1}\right), \cdots, x^{*}\left(x_{n}\right)\right) \in A, x^{*} \in E^{*}\right\}
$$

will be called the Borel cylinder with base $A$ corresponding to $x_{1}, \cdots, x_{n}$. If the elements $x_{1}, \cdots, x_{n}$ generate the linear subspace $M$ of $E$, then we also call the above set a Borel cylinder corresponding to $M$, or a Borel M-cylinder.

The totality of Borel cylinders corresponding to a fixed $M$ form a $\sigma$-algebra, which we denote by $S(M)$, and the totality of all Borel cylinders forms an algebra $S$. Let $\Im$ denote the smallest $\sigma$-algebra containing $S$; we call the elements of $\mathfrak{F}$ weak Borel sets.

Similarly, let $\mathfrak{F}$ be the smallest $\sigma$-algebra of subsets of $E$ which contains all sets of the form

$$
\left\{x \mid x^{*}(x)<a\right\}-\infty<a<\infty, x^{*} \in E^{*} .
$$

The elements of $\mathfrak{F}$ will be called weak Borel sets.

The following lemma shows that the weak Borel sets constitute a sufficiently wide class of sets. 
LEMMA 2.2.1. (c.f. [6])

If $E$ is a separable o-normed space, then every open (or clased) subset of $E$ is a weak Borel set.

LEMMA 2.2.2. (c.f. [6])

Let $E$ be a separable $\sigma$-normed space, with the norm sequence $\left\{\left\|x_{n}\right\|\right\}$. Then, $S_{-n}(R)=\left\{\left\|x^{*}\right\|_{-n} \leqq R\right\}$ is a weak Borel set in $E^{*}$.

By this lemma, we can conclude that $E_{n}^{*}$ is a weak Borel set in $E^{*}$.

Definition 2.2.2. Let $E$ be a linear topological space, and let $S$ be the algebra of all Borel cylinders in $E^{*}$. Suppose that $P$ is a set function on $S$ having the following property: if $M$ is any finite dimensional linear subspace of $E$, and $S(M)$ is the $\sigma$-algebra of Borel cylinders corresponding to $M$, then the restriction of $P$ to $S(M)$ is a probability measure. Then we call $P$ a cylinder measure on $E^{*}$. Clearly, any cylinder measure $P$ also has the following properties:

(1) $o \leqq P(Z) \leqq 1$ for all $Z \in S$

(2) $P\left(E^{*}\right)=1$

(3) $P$ is finitely additive.

However, $P$ is not, in general, $\sigma$-additive.

But if it happens that $P$ is $\sigma$-additive, then, using well-known technique, we can extend $P$ to a probability measure on the $\sigma$-algebra $\mathfrak{F}$ generated by $S$.

Next, we shall show the continuity of cylinder measures.

Definition 2.2.3. Let $E$ be a linear topological space, and let $P$ be a cylinder measure on $E^{*}$. Suppose that, given any positive number $\varepsilon$, there exists a neighborhood $V$ of zero in $E$ such that

$$
P\left(\left\{x^{*}|| x^{*}(x) \mid>1, x^{*} \in E^{*}\right\}\right)<\varepsilon
$$

whenever $x \in V$. Then we say that $P$ is continuous.

LEMma 2.2.3. (c.f. [1], [5])

Let $E$ be a linear topological space and let $P$ be a cylinder measure on $E^{*}$. Then the function

$$
L(x)=\int_{E^{*}} e^{i x^{*}(x)} d P\left(x^{*}\right)
$$

is continuous iff $P$ is continuous.

LEMma 2.2.4. (c.f. [1], [5])

Let $E$ be a linear topological space and $L(x)$ be a continuous positive 
definite function on $E$ [with $L(0)=1]$. Then, there is a unique continuous cylinder measure $P$ on $\left(E^{*}, S\right)$, such that

$$
L(x)=\int_{E^{*}} e^{i x^{*}(x)} d P\left(x^{*}\right) \quad \text { for } \quad x \in E .
$$

Remark. In Lemma 2.2.4, if $E$ is a nuclear space, then $P$ is a probability measure on $\left(E^{*}, \mathfrak{F}\right)$.

If $E$ is a $\sigma$-Hilbert space and $L(x)$ is continuous relative to the nuclear topology, then also $P$ is a probability measure.

(For details, c.f. [1], [5], [6], [7])

\section{$3^{\circ}$. The existence of quasi-invariant measures}

DEFINITION 2.3.1. Let $E$ be a linear space, $\dot{F}$ be a linear subspace of $E$, and let $\mathfrak{B}$ be a $\sigma$-algebra in $E$, which is invariant under translations. $A$ measure $\mu$ on $(E, \mathfrak{B})$ is called $F$-quasi-invariant if

$$
\mu(B)=0 \text { implies } \mu(B+x)=0 \quad \text { for every } x \in F, B \in \mathfrak{B} \text {. }
$$

DEFINITION 2.3.2. Let $E$ be a linear topological space, $E^{*}$ be a adjoint space of $E$, let $\|x\|_{H}$ be a continuous Hilbertian norm on $E$.

It is easily seen that the following $L(x)$ is continuous positive-definite function on $E$.

$$
L(x)=e^{-\frac{\|x\|^{2}}{2}}
$$

The corresponding measure on $E^{*}$ (by Lemma 2.2.4.) is called a Gaussian measure. (mean zero, variance 1 )

Proposition 2.3.1. (c.f. [5[, [8])

Let $E$ be a nuclear space, and $\|x\|_{H}$ be a continuous Hilbertian norm on $E$. Then, the corresponding Gaussian measure $\mu_{H}$ on $E^{*}$ is $\sigma$-additive and E-quasi-invariant.

$$
\left(E \subset H \cong H^{*} \subset E^{*}\right)
$$

Proposition 2.3.2. (c.f. [1], [5], [8])

Let $H, G$ be separable Hilbert spaces such that $G$ is a linear subspace of $H$ and the natural imbedding $(G \rightarrow H)$ is a Hilbert-Schmidt operator.

Let $\mathfrak{F}$ be the $\sigma$-algebra of weak Borel sets in $H$. Then, there exists $a$ (Gaussian) probability measure $\mu$ on $(H, \mathfrak{F})$ which is G-quasi-invariant.

\section{$4^{\circ}$. Dao-Xing's inequality}

In this subsection, we shall show the most important lemma for our purpose. 
Lemma 2. 4. 1. (Dao-Xing's inequality) (c.f. [1], [9])

Let $E$ be a linear topological space, $F$ a linear subspace of $E$, and let $\mathfrak{B}$ be a $\sigma$-algebra in $E$ which is invariant under translations and contains all cylinder sets.

Let $\mathfrak{I}$ be the topology on $F$ such that $(F, \mathfrak{I})$ is a linear topological space of the second category, satisfying the first axiom of countability, and suppose that $\mathfrak{I}$ is stronger than the topology on $F$ induced by $E$.

If there exists a F-quasi-invariant measure $\mu$ on $(E, \mathfrak{B})$, then there exists a neighborhood $V$ of zero in $(F, \mathfrak{T})$ and positive number $C$, such that

$$
\sup _{x \in V}\left|x^{*}(x)\right| \leqq C \int_{E}\left|x^{*}(x)\right| d \mu(x) \quad \text { for every } \quad x^{*} \in E^{*} .
$$

REMARK. In original Dao-Xing's inequality, it was necessary that $\mu$ was locally finite and regular. But in [9], these assumptions were omitted.

\section{§3. Main theorems and other results}

In this section, we shall prove the following main theorems.

Throughout this section, we assume that linear topological spaces are with real coefficients.

Theorem $A$. Let $H$ be a separable Hilbert space, with the inner product $(x, y)$, and let $\mathfrak{F}$ be the totality of weak Borel sets in $H$.

Let $E$ be a linear subspace of $H$, and suppoe that $E$ itself is a complete $\sigma$-normed space with respect to the sequence of norms $\|x\|_{n} n=1,2, \cdots$, where $\|x\|_{1} \leqq\left\|x_{2}\right\| \leqq \cdots$.

Also, suppose that the inclusion mapping $T$ from $E$ into $H$ is continuous. For each $n$, let $E_{n}$ denote the completion of $E$ with respect to the norm $\|x\|_{n}$. Then the following conditions are equivalent.

(1) There exists a E-quasi-invariant finite measure (non-trivial) $\mu$ on $(H, \mathfrak{F})$.

(2) There exists $n$ such that the adjoint operator $T^{*}$ from $H^{*}$ into $E_{n}^{*}$ is absolutely summing.

(3) There exists a separable Hilbert space $H_{1}$ such that

$$
\underset{J}{E \subset H_{1} \subset H}
$$

$T=K \circ J$ where injection map $J$ is continuous and $K$ is a Hilbert-Schmidt operator respectively.

THEOREM $B$. Let $1 \leqq p \leqq 2$, let $\left\{a_{n}\right\}$ be a sequence of positive numbers, and let $l^{p}\left(a_{n}\right)$ denote the totality of real number sequences $\xi=\left\{\xi_{n}\right\}$ which satisfy the condition 


$$
\|\xi\|=\left(\sum_{n=1}^{\infty} a_{n}\left|\xi_{n}\right|^{p}\right)^{\frac{1}{p}}<\infty
$$

$l^{p}\left(a_{n}\right)$ forms a Banach space with respect to the usual coordinatewise linear operations and the norm $\|\xi\|$. In particular, we write $l^{p}$ instead of $l^{p}(1)$. Let $\mathfrak{F}$ be the $\sigma$-algebra in $l^{p}\left(a_{n}\right)$ generated by the totality of Borel cylinders

$$
\left\{\xi \mid\left(\xi_{1}, \xi_{2}, \cdots, \xi_{n}\right) \in B\right\}
$$

(where $B$ represents an arbitrary Borel sets in n-dimensional space).

Let $\Phi$ be a linear subspace of $l^{p}\left(a_{n}\right)$, and suppose that $\Phi$ itself is a complete $\sigma$-Hilbert space with respect to the sequence of inner products $(\varphi, \phi)_{n}, n=1,2, \cdots$ where $(\varphi, \varphi)_{1} \leqq(\varphi, \varphi)_{2} \leqq \cdots$.

Also, suppose that the inclusion mapping $T$ from $\Phi$ into $l^{p}\left(a_{n}\right)$ is continuous. For each $n$, let $\Phi_{n}$ denote the completion of $\Phi$ with respect to the inner product $(\varphi, \psi)_{n}$. Then the following conditions are equivalent.

(1) There exists a $\Phi$-quasi-invariant finite measure (non-trivial) on $\left(l^{p}\left(a_{n}\right), \mathfrak{F}\right)$.

(2) There exists $n_{0}$ such that the adjoint operator $T^{*}$ from $l^{p}\left(a_{n}\right)^{*}$ into $\Phi_{n_{0}}^{*}$ is absolutely summing.

(3) There exists separable Hilbert spaces $H_{1}$ and $H_{2}$ such that

$$
\Phi \subset H_{1} \subset \underset{J}{H_{2} \subset l^{p}}\left(a_{n}\right)
$$

$T=K \circ J \circ I$ where injection map $I$ and $K$ are continuous, $J$ is a HilbertSchmidt operator.

In order to prove these two theorems, the following proposition is necessary.

Proposition 3.1. Let $F$ be a Banach space, $E$ be a linear subspace of $F$, and suppose that $E$ itself is a complete $\sigma$-normed space. Also, suppose that the inclusion mapping $T$ from $E$ into $F$ is continuous.

Then, the existence of a E-quasi-invariant finite measure (non-trivial) $\mu$ on $(F, \mathfrak{F})$ implies that, there exists $n_{0}$ such that
(1) $T^{*}$ is absolutely summing
$\left(T^{*}: \quad F^{*} \rightarrow E_{n_{0}}^{*}\right)$
(2) $T^{*}$ is compact
$\left(T^{*}: F^{*} \rightarrow E_{n_{0}}^{*}\right)$

Proof. (1): We may assume that $\mu$ satisfies the condition

$$
\int_{F}\|x\| d \mu(x)<\infty
$$

for otherwise, we can replace $\mu$ by the equivalent measure 


$$
\mu_{1}(A)=\int_{A} e^{-|x| \mid} d \mu(x) \quad A \in \mathfrak{F}
$$

which certainly satisfies this condition.

Now, by Dao-Xing's inequality, there exists a positive number $C_{1}$ and $n_{0}$ such that

$$
\left\|T^{*}\left(x^{*}\right)\right\|_{E_{n_{0}}^{*}} \leqq C_{1} \int_{F}\left|x^{*}(x)\right| d \mu(x) \quad x^{*} \in F^{*}
$$

Let $\left\{x_{n}^{*}\right\} \subset F^{*}$ be scalarly $l_{1}$, then we may assume that

$$
\sum_{n=1}^{\infty}\left|x_{n}^{*}(x)\right|<\infty \quad x \in F
$$

Putting

$$
p(x)=\sum_{n=1}^{\infty}\left|x_{n}^{*}(x)\right| \quad x \in F,
$$

obviously $p(x)$ is a lower semicontinuous seminorm on $F$.

Since $F$ is a Banach space, using Gelfand's theorem, $p(x)$ is continuous. Therefore, there exists a positive number $C_{2}$ such that

$$
p(x) \leqq C_{2}\|x\| \quad x \in F
$$

Using $(*)$, we get

$$
\begin{aligned}
\sum_{n=\mathrm{I}}^{\infty}\left\|T^{*}\left(x_{n}^{*}\right)\right\|_{E_{n_{0}}^{*}} \leqq C_{1} \int \sum_{n=1}^{\infty}\left|x_{n}^{*}(x)\right| d \mu(x)=C_{1} \int p(x) d \mu(x) \\
\leqq C_{1} C_{2} \int\|x\| d \mu(x)<\infty
\end{aligned}
$$

Q.E.D.

that is the assertion.

(2): Let $\left\{x_{n}^{*}\right\} \subset F^{*}$ be a sequence which converges weakly to zero. Using $(*)$ and Fatou's lemma, we have

$$
\begin{aligned}
& \varlimsup_{n \rightarrow \infty}\left\|T^{*}\left(x_{n}^{*}\right)\right\|_{E_{n_{\rho}}^{*}} \leqq \varlimsup_{n \rightarrow \infty} \int_{F}\left|x_{n}^{*}(x)\right| d \mu(x) \\
&=\int_{F n \rightarrow \infty}\left|x_{n}^{*}(x)\right| d \mu(x)=0 .
\end{aligned}
$$

Namely, $\left\{T^{*}\left(x_{n}^{*}\right)\right\}$ converges strongly to zero in $E_{n_{0}}^{*}$. Therefore, $T^{*}$ is compact.

$$
\text { Q.E.D. }
$$

ExAmple. Identity operator $I: l^{1} \rightarrow l^{\infty}$ is absolutely summing, but it is not compact. Therefore, there is not a $l^{1}$-quasi-invariant finite measure on $\left(l^{\infty}, \mathfrak{F}\right)$. 
Corollary 3.1. Let $E$ be a Banach space, $\mathfrak{B}$ be a $\sigma$-algebra in $E$ which is invariant under translations and contains all cylinder sets.

Then, the following conditions are equivalent.

(1) There exists a E-quasi-invariant finite measure (non-trivial) on $(E, \mathfrak{B})$.

(2) $E$ is finite dimensional.

Proof of Theorem $A$.

$(1) \Rightarrow(2)$ : Using Proposition 3.1., it is obvious.

$(2) \Rightarrow(3)$ : By assumption, there exists $n$ such that the adjoint operator $T^{*}$ from $H^{*}$ into $E_{n}^{*}$ is absolutely summing.

Using Proposition 2.1.2, and Proposition 2.1.6., there exists a Hilbert space $G$ such that

$$
H^{*} \underset{U}{\longrightarrow} G \underset{V}{\longrightarrow} E_{n}^{*}
$$

$T^{*}=V \circ U$ where $U$ is a Hilbert-Schmidt operator and $V$ is a continuous linear operator respectively.

Here, we may assume that $G$ is separable and $U\left(H^{*}\right)$ is dense in $G$. For otherwise, we could replace $G$ by $\overline{U\left(H^{*}\right)}$ (closure of $U\left(H^{*}\right)$ in $G$, which certainly satisfies this condition.

Considering the adjoint operator of $T^{*}=V \circ U$, we get $T^{* *}=U^{*} \circ V^{*}$

$$
E_{n}^{*} \underset{V^{*}}{\longrightarrow} G^{*} \underset{U^{*}}{\longrightarrow} H
$$

Since $U\left(H^{*}\right)$ is dense in $G, U^{*}$ is a injection. Also, since $T=T^{* *}{ }_{\mid E}$, it is easily seen that we have the assetion.

$(3) \Rightarrow(1)$ : Using Proposition 2.3.2, it is obvious.

Proof of Theorem $B$.

$(1) \Rightarrow(2)$ : Using Proposition 3.1., it is obvious.

$(2) \Rightarrow(3)$ : We shall define

$$
\varphi(\xi)=\left(a_{n}^{\frac{1}{p}} \xi_{n}\right) \quad \text { for } \quad \xi=\left(\xi_{n}\right) \in l^{p}\left(a_{n}\right) .
$$

Then, $\varphi(\xi)$ is a linear isometry from $l^{p}\left(a_{n}\right)$ onto $l^{p}$. Therefore, we may assume that $a_{n}=1 \quad n=1,2, \cdots$, .

By assumption, there exists $n_{0}$ such that the adjoint operator $T^{*}$ from $\left(l^{p}\right)^{*}$ into $\Phi_{n_{0}}^{*}$ is absolutely summing. Therefore, by Proposition 2.1.2., it is $p$-absolutely summing. Let $\left\{e_{n}^{*}\right\}$ be a sequence from $\left(l^{p}\right)^{*}$, (where $e_{n}^{*}=\left(\delta_{n k}\right)_{k}$ $\delta_{n k}=\left\{\begin{array}{lll}1 & \text { if } & k=n \\ 0 & \text { if } & k \neq n\end{array}\right)$ then it is easily seen that $\left\{e_{n}^{*}\right\}$ is scalarly $l_{p}$, therefore we get 


$$
\sum_{n=1}^{\infty}\left\|T^{*}\left(e_{n}^{*}\right)\right\|_{\Phi_{n_{0}}^{*} \ldots}^{p}<\infty
$$

Next, we shall show that a linear operator $T$ from $\Phi_{n_{0}}$ into $l^{p}$ is $p$ absolutely summing.

Let $\left\{x_{k}\right\} \subset \Phi_{n_{0}}$ be scalarly $l_{p}$, namely

$$
\sum_{k=1}^{\infty}\left|\left\langle x_{k}, x^{*}\right\rangle\right|^{p}<\infty \quad x^{*} \in \Phi_{n_{0}}^{*},
$$

then, it is easily seen that the following inequality holds

$$
\sup _{\left\|x^{*}\right\| \leq 1} \sum_{k=1}^{\infty}\left|\left\langle x_{k}, x^{*}\right\rangle\right|^{p}<\infty \text {. }
$$

Then,

$$
\begin{aligned}
\sum_{k=1}^{\infty}\left\|T\left(x_{k}\right)\right\|^{p} & =\sum_{k=1}^{\infty} \sum_{n=1}^{\infty}\left|\left\langle T\left(x_{k}\right), e_{n}^{*}\right\rangle\right|^{p}=\sum_{k=1}^{\infty} \sum_{n=1}^{\infty}\left|\left\langle x_{k}, T^{*}\left(e_{n}^{*}\right)\right\rangle\right|^{p} \\
& \leqq\left(\sup _{\left\|x^{*}\right\| \leqq 1} \sum_{k=1}^{\infty}\left|\left\langle x_{k}, x^{*}\right\rangle\right|^{p}\right) \sum_{n=1}^{\infty}\left\|T^{*}\left(e_{n}^{*}\right)\right\|_{\Phi_{n_{0}}^{*}}^{p}<\infty
\end{aligned}
$$

Thus, $T$ is $p$-absolutely summing, and also $T$ is 2 -absolutely summing. By similar discussions for Theorem $A$, we have the assertion.

$(3) \Rightarrow(1)$ : Using Proposition 2.3.2, it is obvious.

Q.E.D.

REMARK (1): Theorem $A$ and Theorem $B$ is the generalization of the Dao-Xing's theorem for complete $\sigma$-normed spaces and $l^{p}\left(a_{n}\right)$-spaces, and also, in Proposition 3.1., if $E$ is a complete $\sigma$-Hilbert space and $F$ is a separable Hilbert space, using Proposition 2.1.5., we get the Dao-Xing's theorem.

Remark (2): In Proposition 3.1., Theorem $A$ and Theorem $B$, if a quasi-invariant measure $\mu$ is $\sigma$-finite, then these results are valid.

Next, we shall show the relations between nuclear spaces and quasiinvariant measures.

LEMMA 3.1. (c. f. [3])

Let $E, F$ and $G$ be Banach spaces respectively, and $T(E \rightarrow F), S(F \rightarrow G)$ be absolutely summing operators respectively.

Then, $S \circ T(E \rightarrow G)$ is nuclear.

PROPOSITION 3.2. Let $E=\bigcap_{n=1}^{\infty} E_{n}$ be a complete o-normed space. Then the following conditions are equivalent.

(1) For any $n$, there exists a E-quasi-invariant finite measure $\mu_{n}$ on $\left(E_{n}, \mathfrak{B}_{n}\right)$. 
(2) E is a nuclear space.

PROOF.

$(1) \Rightarrow(2)$ : Using Proposition 3.1., for any $m$, there exists $n$ such that $T^{*}$ (adjoint operator of the natural injection $T$ ) is an absolutely summing operator from $E_{m}^{*}$ into $E_{n}^{*}$. Furthermore, for $n$, there exists $s$ such that $T^{*}$ is an absolutely summing operator from $E_{n}^{*}$ into $E_{s}^{*}$. Using Proposition 2.1.2., Corollary 2.1.1., Proposition 2.1.4. and Proposition 2.1.5, it is easily. seen that the natural injection $T$ from $E_{s}$ into $E_{m}$ is absolutely summing.

Similarly, there exists $t$ such that the natural injection $T$ from $E_{t}$ into $E_{s}$ is absolutely summing.

Using Lemma 3.1, we have the assertion.

$(2) \Rightarrow(1)$ : Since $E$ is nuclear, hence $E$ is a nuclear $\sigma$-Hilbert space. Thus, we get $E=\bigcap_{n=1}^{\infty} E_{n}=\bigcap_{n=1}^{\infty} \Phi_{n}$ (where $\Phi_{n}$ is a separable Hilbert space).

For any $n$, there exists $s$ and $t$ such that

$$
\Phi_{t} \underset{T}{\longrightarrow} \Phi_{s} \underset{S}{\longrightarrow} E_{n}
$$

(where natural injection $T$ is a Hilbert-Schmidt operator and $S$ is a continuous linear operator respectively.)

Using the similar technique for the proof of Theorem $A$, we have the assertion.

$$
\text { Q.E. D. }
$$

Proposition 3. 3. Let E be a separable o-normed space, and $\mathfrak{F}$ be the totality of weak Borel sets in $E^{*}$. Then the following conditions are equivalent.

(1) For any $n$, there exists a $E_{n}^{*}$-quasi-invariant finite measure $\mu_{n}$ on $\left(E^{*}, \mathfrak{F}\right)$.

(2) E is a nuclear space.

PROOF.

$(1) \Rightarrow(2)$ : Since $E_{s}^{*} \in \mathfrak{\}}$ (see Lemma 2.2.2.), the sequence of sets $E_{s}^{*}$ is monotonic increasing, and $E^{*}=\bigcup_{s=1}^{\infty} E_{s}^{*}$, there exists $s$ such that $\mu_{n}\left(E_{s}^{*}\right)>0$ [with $s \geqq n$ ]. Restricting $\mathfrak{F}$ and $\mu_{n}$ to $E_{s}^{*}$, we obtain a $E_{n}^{*}$-quasi-invariant finite measure $\mu_{n}$ on $\left(E_{s}^{*}, \mathfrak{\Im}_{\mid E_{s}^{*}}\right)$.

Using Proposition 3.1., natural map $\left(E_{s}^{* *} \rightarrow E_{n}^{* *}\right)$ is absolutely summing. Furthermore there exists $t$ such that natural map $\left(E_{t}^{* *} \rightarrow E_{s}^{* *}\right)$ is absolutely summing. Using the same method for the proof of Proposition 3.2, we have the assertion.

$(2) \Rightarrow(1)$ : By the similar discussions for the proof of Proposition 3.2, 
we have the assertion.

$$
\begin{gathered}
\text { EXAMPLE. } \quad L=\bigcap_{n=1}^{\infty} l^{p}\left(a_{m, n}\right) 1 \leqq \\
(m, n=1,2,3, \cdots)
\end{gathered}
$$

$L$ is a complete $\sigma$-normed space with respect to the following norms

$$
\|\xi\|_{n}=\left(\sum_{m=1}^{\infty} a_{m, n}\left|\xi_{m}\right|^{p}\right)^{\frac{1}{p}} \quad \text { for any } \xi=\left(\xi_{m}\right) \in L .
$$

Then, the following conditions are equivalent.

(1) For any $n$, there exists a L-quasi-invariant finite measure $\mu_{n}$ on $\left(l^{p}\left(a_{m, n}\right), \mathfrak{B}_{n}\right)$.

(2) L is nuclear.

(3) For any $n$, there exists s such that

$$
\sum_{m=1}^{\infty} \frac{a_{m, n}}{a_{m, s}}<\infty .
$$

Proof.

$(1) \Leftarrow(2):$ By Proposition 3.2, it is obvious.

$(1) \Rightarrow(3)$ : By Proposition 3.1., there exists $s$ such that the adjoint operator $\left(l^{p}\left(a_{m, n}\right)\right)^{*} \rightarrow\left(l^{p}\left(a_{m, s}\right)\right)^{*}$ is absolutely summing. By easy calculations, we have the assertion.

$(3) \Rightarrow(2)$ : It is easily seen that if for any $n$, there exists $s$ such that

$$
\sum_{m=1}^{\infty}\left(\frac{a_{m, n}}{a_{m, s}}\right)^{\frac{1}{p}}<\infty
$$

then, $L$ is nuclear.

But by assumption, we obtain the followings;

there exists $t, u, \cdots$ such that

$$
\sum_{m=1}^{\infty} \frac{a_{m, s}}{a_{m, t}}<\infty, \quad \sum_{m=1}^{\infty} \frac{a_{m, t}}{a_{m, u}}<\infty \cdots .
$$

Using Hölder's inequality, we have the assertion.

Q.E. D.

Finally, we shall show the relations between Bochner's theorem and quasi-invariant measures.

Proposition 3. 4. (c.f. [7])

Let $\Phi$ be a separable $\sigma$-Hilbert space, with the inner products $\left(\varphi_{1}, \varphi_{2}\right)_{n}^{\phi}$, and let $\Psi$ be a linear subspace of $\Phi$, and suppese that $\Psi$ itself is a complete separable $\sigma$-Hilbert space with respect to the inner products $\left(\psi_{1}, \psi_{2}\right)_{n}^{4}$. Also, suppose that the inclusion mapping $T$ from $\Psi$ into $\Phi$ is continuous. For 
each $n$, let $\Phi_{n},\left(\Psi_{n}\right)$ denote the completion of $\Phi,(\Psi)$ with respect to the inner products $\left(\varphi_{1}, \varphi_{2}\right)_{n}^{\dagger},\left(\left(\psi_{1}, \psi_{2}\right)_{n}^{*}\right)$ respectively. Then, the following conditions are equivalent.

(1) $T$ is a Hilbert-Schmidt operator from $\Psi$ into $\Phi$ in $\sigma$-Hilbert spaces. Namely, for any $m$, there exists $n$ such that $T$ is a Hilbert-Schmidt operator from $\Psi_{n}$ into $\Phi_{m}$.

(2) For any $n$, there exists a $\Psi$-quasi-invariant finite measure $\mu_{n}$ on $\left(\Phi_{n}, \mathfrak{B}_{n}\right)$.

(3) For any continuous cylinder set measure $\mu$ in $\Phi^{*}$, the cylinder set measure $T^{*} \mu$ in $\Psi^{*}$ induced by $T$ and $\mu$ is $\sigma$-additive.

(4) Let $\mu_{n}$ be the Gaussian measure, defined in $\Phi^{*}$ by $\left(\varphi_{1}, \varphi_{2}\right)_{n}^{\oplus}$, then for any $n$, the measure $T^{*} \mu_{n}$ in $\Psi^{*}$ induced by $T$ and $\mu_{n}$ is $\sigma$-additive.

(5) For any positive definite continuous function $L(\varphi)$ on $\Phi$ with $L(0)=1$, there exists a unique probability measure $\mu$ on $\left(\Psi^{*}, \mathfrak{F}\right)$ such that

$$
L(\phi)=\int_{\psi *} e^{i F(\psi)} d(F)
$$

ProOF.

$(1) \Rightarrow(2):$ For any $n$, there exists $s$ such that $T$ is a Hilbert-Schmidt operator from $\Psi_{s}$ into $\Phi_{n}$.

Using the similar method for the proof of Theorem $A$, there exists a separable Hilbert space $H$ such that

$$
\Psi \underset{S}{\stackrel{S}{\leftrightarrows}} \underset{\vec{U}}{\subsetneq} \Phi_{n}
$$

$T=U \circ S$ where the injection map $S$ is a continuous linear operatar and $U$ is a Hilbert-Schmidt operator respectively.

Using Proposition 2.2.3., we have the assertion.

$(2) \Rightarrow(1)$ : Using Proposition 3.1. and Proposition 3.1.5., it is obvious.

Finally, by [7], (1), (3) and (4) are equivalent, and by Lemma 2.2.3. and Lemma 2.2.4., (3) and (5) are equivalent. Thus we have the conclusion.

Q.E.D.

Remark (1) In the above Proposition, $\boldsymbol{\sigma}$-algebra $\mathfrak{B}_{n}(\mathfrak{F})$ are weak Borel sets in $\Phi_{n}\left(\Psi^{*}\right)$ respectively.

Remark (2) In the above Proposition, let $\Phi$ and $\Psi$ be separable Hilbert spaces, then the Dao-Xing's results is that (1), (3) and (4) are equivalent.

§4. Further discussions for $l^{p}\left(a_{n}\right)$ and $L^{p}(X, \mu)$

Throughout this section, we assume that linear spaces are with real coefficients. 
$1^{\circ}$. Let $1 \leqq q<\infty$, let $\left\{b_{m, n}\right\}$ be a double sequence of positive numbers with

$$
b_{m, n} \leqq b_{m+1, n} \quad m, n=1,2, \cdots
$$

and let $\bigcap_{m=1}^{\infty} l^{q}\left(b_{m, n}\right)$ denote the totality of real number sequences $\xi=\left(\xi_{n}\right)$ which satisfies the condition

$$
\|\xi\|_{m}=\left(\sum_{n=1}^{\infty} b_{m, n}\left|\xi_{n}\right|^{q}\right)^{\frac{1}{q}}<\infty \quad m=1,2, \cdots
$$

then, $\bigcap_{m=1}^{\infty} l^{q}\left(b_{m, n}\right)$ forms a complete $\sigma$-normed space with respect to the usual coordinatewise linear operations and norms $\|\xi\|_{m}$. Let $l^{p}\left(a_{n}\right)$ and $\mathfrak{F}$ be the same notations of Theorem $B$.

Then, we have the followings.

PROPOSITION 4.1.1. Let $\bigcap_{m=1}^{\infty} l^{q}\left(b_{m, n}\right)$ be a linear subspace of $l^{p}\left(a_{n}\right)$ and let the injection map $T: \bigcap_{m=1}^{\infty} l^{q}\left(b_{m, n}\right) \rightarrow l^{p}\left(a_{n}\right)$ be continuous. If we also assume that $1 \leqq p \leqq 2,1 \leqq q \leqq 2$, then the following conditions are equivalent.

(1) There exists a $\bigcap_{m=1}^{\infty} l^{q}\left(b_{m, n}\right)$-quasi-invariant finite measure on $\left(l^{p}\left(a_{n}\right), \mathfrak{F}\right)$.

(2) There exists $m_{0}$ such that the adjoint operator $T^{*}: l^{p}\left(a_{n}\right)^{*} \rightarrow l^{q}\left(b_{m_{0}, n}\right)^{*}$ is absolutely summing.

(3) There exists $m_{0}$ such that the adjoint operator $T^{*}: l^{p}\left(a_{n}\right)^{*} \rightarrow l^{q}\left(b_{m_{0}, n}\right)^{*}$ is p-absolutely summing.

(4) There exists $m_{0}$ such that

$$
\sum_{n=1}^{\infty} a_{n} / b_{m_{0}, n}^{\frac{p}{q}}<\infty .
$$

(5) There exist separable Hilbert space $H_{1}$ and $H_{2}$ such that

$$
\bigcap_{m=1}^{\infty} l^{q}\left(b_{m, n}\right) \underset{I}{\leftrightarrows} H_{1} \underset{J}{\subsetneq} H_{2} \underset{K}{\leftrightarrows} l^{p}\left(a_{n}\right)
$$

$T=K \circ J \circ I$ where injection map $I$ and $K$ are continuous, and $J$ is a HilbertSchmidt operator.

Proof. Using Proposition 2.1.2, Proposition 2.3.2, and Proposition 3.1., $(1) \Rightarrow(2) \Rightarrow(3)$ and $(5) \Rightarrow(1)$ are obvious.

$(4) \Rightarrow(5)$ : We assume that there exists $m_{0}$ such that

$$
\sum_{n=1}^{\infty} a_{n} / b_{m_{0}, n}^{\frac{p}{q}}<\infty
$$

then we have 


$$
l^{a} \underset{I}{\subsetneq} l^{2} \underset{J}{\subsetneq} l^{2}\left(a_{n} / b_{m_{0}, n}^{\frac{p}{q}}\right) \underset{K}{\leftrightarrows} l^{p}\left(a_{n} / b_{m_{0}, n}^{\frac{p}{q}}\right)
$$

where natural injections $I$ and $K$ are continuous, and $J$ is a Hilbert-Schmidt operator.

Next, we shall consider $\varphi(\xi)=\left(b_{m_{0}, n}^{\frac{1}{q}} \xi_{n}\right)$ for $\xi=\left(\xi_{n}\right) \in l^{p}\left(a_{n}\right)$, then it is clear that $l_{p}\left(a_{n}\right)$ and $l^{p}\left(a_{n} / b_{m_{0}, n}^{\frac{p}{q}}\right)$ are linearly isometric by $\varphi(\xi)$. Thus we have

$$
\begin{aligned}
\bigcap_{m=1}^{\infty} l^{q}\left(b_{m, n}\right) & \subset l^{q}\left(b_{m_{0}, n}\right)=\varphi^{-1}\left(l^{q}\right) \\
& \subset \varphi^{-1}\left(l^{2}\right) \subset \varphi^{-1}\left(l^{2}\left(a_{n} / b_{m_{0}, n}^{\frac{p}{q}}\right)\right) \\
& \subset \varphi^{-1}\left(l^{p}\left(a_{n} / b_{m_{0}, n}^{\frac{p}{q}}\right)\right)=l^{p}\left(a_{n}\right) .
\end{aligned}
$$

Putting $H_{1}=\varphi^{-1}\left(l^{2}\right), H_{2}=\varphi^{-1}\left(l^{2}\left(a_{n} / b_{m_{0}, n}^{\frac{p}{q}}\right)\right)$, we have the assertion.

Finally, for $(3) \Rightarrow(4)$, we shall prove more general case in $\S 4.2^{\circ}$. Proposition 4.2.1..

$$
\text { Q.E.D. }
$$

REMARK. If $1 \leqq p<\infty, 1 \leqq q \leqq 2$, then we can show that (1), (2), (3) and (4) are equivalent, and (5) $\Rightarrow(1)$ is valid, however, $(4) \Rightarrow(5)$ is not valid. Furthermore, if $1 \leqq p<\infty, 1 \leqq q<\infty$, then, $(1) \Rightarrow(2) \Rightarrow(3) \Rightarrow(4)$ are valid, however, (4) $\Rightarrow(1)$ is not valid. (c.f. Proposition 4.1.2., Proposition 4.1.3.)

Proposition 4.1.2. Let $2 \leqq p<\infty, 1<q<\infty$, and $l^{a} \subset l^{p}\left(a_{n}\right)$, $\left[\right.$ with $\left.\sum_{n=1}^{\infty} a_{n}<\infty\right]$. If there exists a $l^{q}$-quasi-invariant finite measure $\mu$ on $\left(l^{p}\left(a_{n}\right), \mathfrak{F}\right)$

Then, we have

$$
\sum_{n=1}^{\infty} a_{n}^{\frac{g^{*}}{2}}<\infty \quad\left(\frac{1}{q}+\frac{1}{q^{*}}=1\right)
$$

Proof. Since $l^{p}\left(a_{n}\right) \subset l^{2}\left(a_{n}\right)$, by assumption, we can easily show that there exists $l^{a}$-quasi-invariant finite measure $\mu$ on $\left(l^{2}\left(a_{n}\right), \mathfrak{F}\right)$.

Then, by Theorem $A$, injection map $\left(l^{a} \rightarrow l^{2}\left(a_{n}\right)\right)$ is a Hilbert-Schmidt operator, theorefore it is $q^{*}$-absolutely summing.

From this, we have easily the assertion.

Q.E. D.

Proposition 4. 1.3. Let $1 \leqq p<\infty, 1<q<\infty$, and $l^{q} \subset l^{p}\left(a_{n}\right)$, where the injection map is continuous. If the injection map is a Hilbert-Schmidt operator, then we have 


$$
\sum_{n=1}^{\infty} a_{n}^{\frac{q^{*}}{p}}<\infty
$$

Proof is easy.

$2^{\circ}$. Throughout this subsection, let $X$ be a set and $\mathfrak{B}$ be a $\sigma$-algebra in $X$, and let $\mu$ and $\nu$ be non-trivial positive measure on $(X, \mathfrak{B})$.

Proposition 4.2.1. Let $L^{q}(X, \nu) \subset L^{p}(X, \mu)(1 \leqq p<\infty, 1 \leqq q<\infty)$ be usual Banach spaces and let the injection map $T\left(L^{q}(X, \nu) \rightarrow L^{p}(X, \mu)\right)$ be continuous. Let $\mathfrak{B}_{p}$ be a $\sigma$-algebra in $L^{p}(X, \mu)$ which is invariant under translations and contains all cylinder sets.

Then, the following implications $(1) \Rightarrow(2) \Rightarrow(3) \Rightarrow(4)$ holds.

(1) There exists a $L^{q}(X, \nu)$-quasi-invariant finite measure on $\left(L^{p}(X, \mu), \mathfrak{B}_{p}\right)$.

(2) The adjoint operator $T^{*}: L^{p}(X, \mu)^{*} \rightarrow L^{q}(X, \nu)^{*}$ is absolutely summing.

(3) The adjoint operator $T^{*}: L^{p}(X, \mu)^{*} \rightarrow L^{q}(X, \nu)^{*}$ is $p$-absolutely summing.

(4) For any $\left\{X_{n}\right\} \subset X$ which is measurable and pairwise disjoint with $0<\mu\left(X_{n}\right)<\infty, 0<\nu\left(X_{n}\right)<\infty$,

we have

$$
\sum_{n=1}^{\infty} \frac{\mu\left(X_{n}\right)}{\nu\left(X_{n}\right)^{\frac{p}{q}}}<\infty .
$$

Proof. The implicatios $(1) \Rightarrow(2) \Rightarrow(3)$ are valid by Proposition 3.1. and Proposition 2.1.2.

$(3) \Rightarrow(4)$ : We shall define

$$
f_{n}(x)=\left\{\begin{array}{cc}
\mu\left(X_{n}\right)^{\frac{1}{p}-1} & \text { for } x \in X_{n} \\
0 & \text { for } x \in X_{n}^{c},
\end{array}\right.
$$

then $\left\{f_{n}\right\} \subset L^{p}(X, \mu)^{*}$ is scalarly $l_{p}$ by the following $(*)$.

(*) If $p=1$, then for any $g \in L^{\infty}(X, \mu)^{*}$, there exist complex sequence $\left\{\alpha_{n}\right\}$ such that $\left|\alpha_{n}\right|=1,\left|\left\langle f_{n}, g\right\rangle\right|=\alpha_{n}\left\langle f_{n}, g\right\rangle$.

Therefore, for any positive integer $N$, we have

$$
\begin{aligned}
\sum_{n=1}^{N}\left|\left\langle f_{n}, g\right\rangle\right| & =\left\langle\sum_{n=1}^{N} \alpha_{n} f_{n}, g\right\rangle \\
& \leqq\left\|\sum_{n=1}^{N} \alpha_{n} f_{n}\right\|_{L_{\infty}}\|g\|_{L_{\infty}^{*}} \leqq\|g\|_{L_{\infty}^{*}} .
\end{aligned}
$$

Thus, we have the assertion.

If $p>1$, then for any $g \in L^{p}(X, \mu)$, we have 


$$
\begin{aligned}
\left|\left\langle f_{n}, g\right\rangle\right| & =\left|\int_{X_{n}} f_{n}(x) g(x) d \mu(x)\right| \\
& \leqq\left[\int_{X_{n}}\left|f_{n}(x)\right|^{p^{*}} d \mu(x)\right]^{\frac{1}{p^{*}}}\left[\int_{X_{n}}|g(x)|^{p} d \mu(x)\right]^{\frac{1}{p}} \\
& =\left[\int_{X_{n}}|g(x)|^{p} d \mu(x)\right]^{\frac{1}{p}} .
\end{aligned}
$$

From this, we have

$$
\begin{aligned}
\sum_{n=1}^{\infty}\left|\left\langle f_{n}, g\right\rangle\right|^{p} & \leqq \sum_{n=1}^{\infty} \int_{x_{n}}|g(x)|^{p} d \mu(x) \\
& \leqq \int_{x}|g(x)|^{p} d \mu(x)<\infty .
\end{aligned}
$$

That is the assertion.

Next, by assumption, the adjoint operator $T^{*}: L^{p}(X, \mu)^{*} \rightarrow L^{q}(x, \nu)^{*}$ is $p$-absolutely summing, namely

$$
\sum_{n=1}^{\infty}\left\|T^{*}\left(f_{n}\right)\right\|_{\left.L^{(}\right)^{*}}^{p}<\infty
$$

On the other hand,

$$
\begin{aligned}
\left\|T^{*}\left(f_{n}\right)\right\|_{\left(L^{q}\right)^{*}} & =\sup _{\|\cdot\|_{I} q \leq 1}\left|\left\langle T^{*}\left(f_{n}\right), g\right\rangle\right| \\
& =\sup _{\|\cdot\|_{I} \|^{q \leq 1}}\left|\left\langle f_{n}, T(g)\right\rangle\right|=\sup _{\|\cdot\|_{L^{q}}}\left|\int f_{n} g d \mu\right| \\
& \geqq \mu\left(X_{n}\right) \mu\left(X_{n}\right)^{\frac{1}{p}-1}\left[\frac{1}{\nu\left(X_{n}\right)}\right]^{\frac{1}{q}}=\frac{\mu\left(X_{n}\right)^{\frac{1}{p}}}{\nu\left(X_{n}\right)^{\frac{1}{q}}} .
\end{aligned}
$$

Thus, we have

$$
\sum_{n=1}^{\infty} \frac{\mu\left(X_{n}\right)}{\nu\left(X_{n}\right)^{\frac{p}{q}}} \leqq \sum_{n=1}^{\infty}\left\|T^{*}\left(f_{n}\right)\right\|_{\left(L^{q}\right)^{*}}^{p}<\infty .
$$

That is the conclusion.

Q.E.D.

Let $(X, \mathfrak{B}, \mu)$ be a measure space. The $\mu$-measurable set $E$ of positive measure is called an atom whenever for any $\mu$-measurable subset $E_{1}$ of $E$ we have either $\mu\left(E_{1}\right)=0$ or $\mu\left(E-E_{1}\right)=0$.

If $(X, \mathfrak{B}, \mu)$ be a $\sigma$-finite measure space, then we may show that $X=$ $X_{1}+X_{2}$ uniquely, where neither $X_{1}$ nor any of its measurable subsets is an atom, and $X_{2}$ is a union of an at most countable number of atoms of finite measure. When this, we shall say $X_{1}$ non atomic part of $\mu$. 
THEOREM 4.2.1. Let $(X, \mathfrak{B}, \mu)$ be a non-trivial finite measure space, and let $\mathfrak{B}_{p}$ be a $\sigma$-algebra in $L^{p}(X, \mu)$ which is invariant under translations and contains all cylinder sets. If $1 \leqq p \leqq q \leqq 2$, then the following condition are equivalent.

(1) There exists a $L^{q}(X, \mu)$-quasi-invariant finite measure (non-trivial on $\left(L^{p}(X, \mu), \mathfrak{B}_{p}\right)$.

(2) The adjoint operator $\left(L^{p}(X, \mu)^{*} \rightarrow L^{q}(X, \mu)^{*}\right)$ is absolutely summing.

(3) The adjoint operator $\left(L^{p}(X, \mu)^{*} \rightarrow L^{q}(X, \mu)^{*}\right)$ is p-absolutely summing.

(4) For any $\left\{X_{n}\right\} \subset X$ which is measurablt and pairwise disjoint, we have $\sum_{n=1}^{\infty} \mu\left(X_{n}\right)^{1-\frac{p}{q}}<\infty$.

PRoof. The implications. $(1) \Rightarrow(2) \Rightarrow(3) \Rightarrow(4)$ are valid by Proposition 3.1., Proposition 2.1.2, and Proposition 4.2.1.

$(4) \Rightarrow(1)$ : Suppose that the condition (4), then it is easily seen that the non-atomic part of $\mu$ has zero measure.

Since $\mu(X)<\infty, \mu$ is concentrated on at most countable sets.

In this case, using Proposition 4.1.1, we have the assertion.

Q.E.D.

REMARK. If $1 \leqq p \leqq q<\infty$, the implications (1) $\Rightarrow(2) \Rightarrow(3) \Rightarrow(4)$ are valid. However, $(4) \Rightarrow(1)$ is not valid.

Corollary 4.2.1. Let $1 \leqq p \leqq q<\infty$, and let $(X, \mathfrak{B}, \mu)$ be a finite measure space. If the non-atomic part of $\mu$ has a positive measure, then there exist no $L^{q}(X, \mu)$-quasi-invariant finite measure on $\left(L^{p}(X, \mu), \mathfrak{B}_{p}\right)$.

ExAmple. Let $\mu$ be a Lebesgue measure on $([a, b], \mathfrak{B})$, and let $1 \leqq p \leqq$ $q<\infty$. Then there exist no $L^{q}(X, \mu)$-quasi-invariant finite measure on $\left(L^{p}(X, \mu), \mathfrak{B}_{p}\right)$.

Proposition 4.2.2. Let $(X, \mathfrak{B}, \mu)$ be a $\sigma$-finite measure space, and $\mathfrak{B}_{p}$ be a $\sigma$-algebra in $L^{p}(X, \mu)$ which is invariant under translations and contains all cylinder sets. If the non-atomic part of $\mu$ has a positive measure, then there exist no $L^{p}(X, \mu) \cap L^{q}(X, \mu)$-quasi-invariant finite measure on $\left(L^{p}(X, \mu), \mathfrak{B}_{p}\right)$, for any $1 \leqq p \leqq \infty, 1 \leqq q \leqq \infty$.

PROOF. Let $X_{1}$ be a non-atomic part of $\mu$.

Case 1. $0<\mu\left(X_{1}\right)<\infty$.

Assume the contrary, then it is easily seen that there exists $L^{p}\left(X_{1}, \mu\right) \cap$ $L^{q}\left(X_{1}, \mu\right)$-quasi-invariant finite measure on $\left(L^{p}\left(X_{1}, \mu\right), \mathfrak{B}_{p}^{1}\right)$, but by Corollary 4.2.1., it is a contradiction.

Case 2. $\mu\left(X_{1}\right)=\infty$.

Then, there exists $\left\{X^{(n)}\right\} \subset X_{1}$ which is measurable and pairwise disjoint 
with

$$
\mu\left(X^{(n)}\right)=1 \quad n=1,2, \cdots
$$

We shall define

$$
f_{n}(x)= \begin{cases}1 & \text { for } \quad x \in X^{(n)} \\ 0 & \text { for } \left.x \in X^{(n) c} \quad \text { (complement of } X^{(n)}\right) .\end{cases}
$$

Then, we have $\left\{f_{n}\right\} \subset L^{p}(X, \mu) \cap L^{q}(X, \mu)$.

Since $L^{p}(X, \mu) \cap L^{q}(X, \mu)$ is a Banach space with the norm $\|f\|=\|f\|_{p}$ $+\|f\|_{q}$ for $f \in L^{p}(X, \mu) \cap L^{q}(X, \mu)$, and natural injection $\left(L^{p}(X, \mu) \cap L^{q}(X, \mu) \rightarrow\right.$ $\left.L^{p}(X, \mu)\right)$ is continuous, if we assume the contrary, by Proposition 3.1., natural injection $\left(L^{p}(X, \mu) \cap L^{q}(X, \mu) \rightarrow L^{p}(X, \mu)\right)$ must be compact.

However, $\left\{f_{n}\right\}$ is bounded in $L^{p}(X, \mu) \cap L^{q}(X, \mu)$ and

$$
\left\|f_{n}-f_{m}\right\|_{p}=\left\{\begin{array}{ll}
2^{\frac{1}{p}} & \text { for } 1 \leqq p<\infty \\
1 & \text { for } p=\infty
\end{array} \text { for any } n \neq m,\right.
$$

therefore, any subsequence of $\left\{f_{n}\right\}$ is not convergent in $L^{p}(X, \mu)$.

That is a contradiction.

Q. E.D.

REMARK. In the above Proposition, we may take the following condition $(*)$ instead of the above condition.

(*) There exist positive number $C_{1}, C_{2}$ and $\left\{X_{n}\right\} \subset X$ which is measurable and pairwise disjoint such that

$$
C_{1} \leqq \mu\left(X_{n}\right) \leqq C_{2} \quad n=1,2, \cdots .
$$

EXAMPLE. Let $\mu$ be a Lebesgue measure on $\left(R^{N}, \mathfrak{B}\right)$, then there exist no $L^{p}\left(R^{N}, \mu\right) \cap L^{q}\left(R^{N}, \mu\right)$-quasi-invariant finite measure on $\left(L^{p}\left(R^{N}, \mu\right), \mathfrak{B}_{p}\right)$, for $1 \leqq p \leqq \infty, 1 \leqq q \leqq \infty$.

\section{§5. Appendix}

Let $H_{1}$ and $H_{2}$ be Hilbert spaces. The class of all Hilbert-Schmidt operators from $H_{1}$ into $H_{2}$ (resp. of all absolutely $p$-summing operators) will be denote by $\mathfrak{S}_{2}\left(H_{1}, H_{2}\right)$ (resp. $\Pi_{p}\left(H_{1}, H_{2}\right)$ ).

Then, using a quasi-invariant measure, we shall give another proof of the following Pietsch's Theorem.

Theorem. $\mathfrak{\Im}_{2}\left(H_{1}, H_{2}\right)=\Pi_{p}\left(H_{1}, H_{2}\right)$ for $1 \leqq p \leqq 2$.

First, we shall prove the following Lemma.

LEMMA 5.1. : (c. f. [3])

Let $E_{1}$ and $E_{2}$ be normed sprces. If $1 \leqq p \leqq q<\infty$, then 


$$
\Pi_{p}\left(E_{1}, E_{2}\right) \subset \Pi_{q}\left(E_{1}, E_{2}\right) .
$$

PRoof. We may assume that $1 \leqq p<q<\infty$.

If $T \in \Pi_{p}\left(E_{1}, E_{2}\right)$, then for each $\left\{x_{n}\right\} \subset E_{1}$ which is scalarly $l_{q}$, and for each $\left\{\lambda_{n}\right\} \in l_{q / q-p}$ and $x^{*} \in E_{1}^{*}$ (dual of $E_{1}$ ), we have

$$
\begin{aligned}
& \sum_{n=1}^{\infty}\left|\left\langle\left|\lambda_{n}\right|^{\frac{1}{p^{n}}} x_{n}, x^{*}\right\rangle\right|^{p}=\sum_{n=1}^{\infty}\left|\lambda_{n}\right|\left|\left\langle x_{n}, x^{*}\right\rangle\right|^{p} \\
& \leqq\left(\sum_{n=1}^{\infty}\left|\lambda_{n}\right|^{q / q-p}\right)^{q-p / q}\left(\sum_{n=1}^{\infty}\left|\left\langle x_{n}, x^{*}\right\rangle\right|^{q}\right)^{\frac{p}{q}}<\infty .
\end{aligned}
$$

Namely, $\left\{\left|\lambda_{n}\right|^{\frac{1}{p}} x_{n}\right\} \subset E_{1}$ is scalarly $l_{p}$, therefore we have

$$
\sum_{n=1}^{\infty}\left\|T\left(\left|\lambda_{n}\right|^{\frac{1}{p}} x_{n}\right)\right\|^{p}=\sum_{n=1}^{\infty}\left|\lambda_{n}\right|\left\|T\left(x_{n}\right)\right\|^{p}<\infty .
$$

Thus, we have

$$
\left\{\left\|T\left(x_{n}\right)\right\|^{p}\right\} \in l_{q / p} \quad\left(\text { dual of } l_{q / q-p}\right) .
$$

From this, we have easily the conclusion.

Lemma 5. 2. $\mathfrak{S}_{2}\left(H_{1}, H_{2}\right) \subset \Pi_{1}\left(H_{1}, H_{2}\right)$

Q.E.D.

PROOF.

Case 1. Let $H_{1}$ and $H_{2}$ be separable Hilbert spaces with real coefficients. If $T \in \mathfrak{S}_{2}\left(H_{1}, H_{2}\right)$, then we have the following decomposition :

$$
H_{1} \stackrel{T_{1}}{\longrightarrow} \overline{T\left(H_{1}\right)} \stackrel{I}{\longrightarrow} H_{2} \quad T=I \circ T_{1}
$$

where $\overline{T\left(H_{1}\right)}$ is the closure of $T\left(H_{1}\right)$ in $H_{2}, T_{1}(=T)$ is the Hilbert-Schmidt operator from $H_{1}$ into $\overline{T\left(H_{1}\right)}$ and $I$ is the identity map from $\overline{T\left(H_{1}\right)}$ into $H_{2}$.

Since the image of $H_{1}$ by $T_{1}$ is dense in $\overline{T\left(H_{1}\right)}, T_{1}^{*}$ (adjoint operator of $T_{1}$ ) is a injection map from $\overline{T\left(H_{1}\right)^{*}}$ (dual of $\overline{T\left(H_{1}\right)}$ ) into $H_{1}^{*}$ and also a Hilbert-Schmidt operator.

Then, we may consider that $\overline{T\left(H_{1}\right)^{*}}$ is a linear subspace of $H_{1}^{*}$ and natural injection is a Hilbert-Schmidt operator.

Therefore, by Proposition 2.3.2, there exists a $\overline{T\left(H_{1}\right)}{ }^{*}$-quasi-invariant Gaussian measure $\mu$ on $\left(H_{1}^{*}, \mathfrak{F}\right)$.

Hence, by Proposition 3.1., $T_{1}^{* *}$ (adjoint operator of $T_{1}^{*}$ ) is a absolutely summing operator from $H_{1}$ into $\overline{T\left(H_{1}\right)}$.

From this and $T_{1}^{* *}=T_{1}$, we have easily the conclusion.

Case 2. Let $H_{1}$ and $H_{2}$ be separable Hilbert spaces with complex coefficients. 
Since $H_{1}$ and $H_{2}$ are isomorphic to $l^{2}$ (usual Hilbert space with complex coefficient), we may assume that $H_{1}=H_{2}=l^{2}$.

For any $\xi=\left(\xi_{n}\right) \in l^{2}$, we shall denote $\left(\operatorname{Re} . \xi_{n}\right)$ [resp. (Im. $\left.\left(\xi_{n}\right)\right]$ by Re. $\xi$ [resp. Im. $\xi$ ], then it is easily seen that Re. $\xi \in l_{R}^{2}$ (usual Hilbert space with real coefficient) and $\operatorname{Im} . \xi \in l_{R}^{2}$.

Let $\left\{x_{n}\right\} \subset l^{2}$ be scalarly $l_{1}$, then by the following $(*),\left\{\right.$ Re. $\left.x_{n}\right\} \subset l_{R}^{2}$ and $\left\{\right.$ Im. $\left.x_{n}\right\} \subset l_{R}^{2}$ are scalarly $l_{1}$ respectively.

$(*)$ By assumptions, for any $x=\left(\zeta_{k}\right) \in l_{R}^{2}$

we have

$$
\sum_{n=1}^{\infty}\left|\left\langle x_{n}, x\right\rangle\right|<\infty
$$

Let Re. $x_{n}=\left(\xi_{k}^{(n)}\right)$ and $\operatorname{Im} . x_{n}=\left(\eta_{k}^{(n)}\right)$, then

$$
\sum_{n=1}^{\infty}\left|\sum_{k=1}^{\infty} \xi_{k}^{(n)} \zeta_{k}+i \sum_{k=1}^{\infty} \eta_{k}^{(n)} \zeta_{k}\right|<\infty
$$

hence we have

$$
\sum_{n=1}^{\infty}\left|\sum_{k=1}^{\infty} \xi_{k}^{(n)} \zeta_{k}\right|<\infty, \quad \sum_{n=1}^{\infty}\left|\sum_{k=1}^{\infty} \eta_{k}^{(n)} \zeta_{k}\right|<\infty
$$

Namely,

$$
\sum_{n=1}^{\infty}\left|\left\langle\operatorname{Re} . x_{n}, x\right\rangle\right|<\infty, \quad \sum_{n=1}^{\infty}\left|\left\langle\operatorname{Im} . x_{n}, x\right\rangle\right|<\infty .
$$

Now, let $T \in \mathscr{S}_{2}\left(H_{1}, H_{2}\right)$, since

$$
\begin{aligned}
T\left(x_{n}\right) & =T\left(\operatorname{Re} . x_{n}\right)+i T\left(\operatorname{Im} . x_{n}\right) \\
& =\operatorname{Re} . T\left(\operatorname{Re} . x_{n}\right)+i \operatorname{Im} . T\left(\operatorname{Re} . x_{n}\right)+\operatorname{Re} . i T\left(\operatorname{Im} . x_{n}\right)+i \operatorname{Im} . i T\left(\operatorname{Im} . x_{n}\right),
\end{aligned}
$$

it is sufficient to show that:

$$
\begin{aligned}
& \sum_{n=1}^{\infty}\left\|\operatorname{Re} . T\left(\operatorname{Re} . x_{n}\right)\right\|<\infty, \quad \sum_{n=1}^{\infty}\left\|\operatorname{Im} . T\left(\operatorname{Re} . x_{n}\right)\right\|<\infty, \\
& \sum_{n=1}^{\infty}\left\|\operatorname{Re} . i T\left(\operatorname{Im} . x_{n}\right)\right\|<\infty, \quad \sum_{n=1}^{\infty}\left\|\operatorname{Im} . i T\left(\operatorname{Im} . x_{n}\right)\right\|<\infty .
\end{aligned}
$$

Let $J$ be a continuous linear mapping from $l^{2}$ into $l_{R}^{2}$ such that $J(x)=R e . x$ for any $x \in l^{2}$, then $J \circ T$ is a Hilbert-Schmidt operator from $l_{R}^{2}$ into $l_{R}^{2}$.

Using Case $1 ., J \circ T$ is a absolutely summing operator from $l_{R}^{2}$ into $l_{R}^{2}$, and therefore we have

$$
\sum_{n=1}^{\infty}\left\|\operatorname{Re} . T\left(\operatorname{Re} . x_{n}\right)\right\|<\infty .
$$


By similar arguments, we have the assertion.

Finally, we shall prove the general case.

Let $T \in \mathfrak{S}_{2}\left(H_{1}, H_{2}\right)$, for any $\left\{x_{n}\right\} \subset H_{1}$ which is scalarly $l_{1}$, we shall denote a closed linear subspace of $H_{1}$ generated by $\left\{x_{n}\right\}$ by $M_{1}$ and denote the closure of $T\left(M_{1}\right)$ in $H_{2}$ by $M_{2}$, then $T$ is a Hilbert-Schmidt operator from $M_{1}$ into $M_{2}$, and $\left\{x_{n}\right\} \subset M_{1}$ is scalarly $l_{1}$, and therefore using Case 2., we have

$$
\sum_{n=1}^{\infty}\left\|T\left(x_{n}\right)\right\|<\infty \text {. }
$$

That is the assertion.

$$
\text { Q.E.D. }
$$

Proof of Theorem. By Lemma 5.1. and Lemma 5.2, we have

$$
\begin{array}{r}
\Im_{2}\left(H_{1}, H_{2}\right) \subset \Pi_{p}\left(H_{1}, H_{2}\right), \quad \Pi_{p}\left(H_{1}, H_{2}\right) \subset \Pi_{2}\left(H_{1}, H_{2}\right) \\
\text { for } 1 \leqq p \leqq 2 .
\end{array}
$$

Therefore, it is sufficient to show that $\Pi_{2}\left(H_{1}, H_{2}\right) \subset \mathfrak{S}_{2}\left(H_{1}, H_{2}\right)$.

If $T \in \Pi_{2}\left(H_{1}, H_{2}\right)$, for any orthonormal sequence $\left\{e_{n}\right\} \subset H_{1}$, since $\left\{e_{n}\right\} \subset H_{1}$ is scalarly $l_{2}$, we have

$$
\sum_{n=1}^{\infty}\left\|T\left(e_{n}\right)\right\|^{2}<\infty
$$

Namely, $T$ is a Hilbert-Schmidt operator from $H_{1}$ into $H_{2}$.

Q.E. D.

Department of Mathematics

Hokkaido University

\section{References}

[1] Xia DAO-XING: Measure and integration theory on infinite-dimensional spaces, Academic Press New York (1972).

[2] A. Pietsch : Nuclear Locally Convex Spaces, Springer-Verlag Berlin New York (1972).

[ 3 ] A. Pietsch : Absolut p-summierende Abbildungen in normierten Räumen, Studia Math. 28 (1967), p. 333-353.

[4] A. PelczYŃSKI : A characterization of Hilbert-Schmidt operators, Studia Math. 28 (1967).

[5] I. M. Gelfand and N. J. Vilenkin: Generalized Functions, Vol. 4 (1961).

[6] R. A. Minlos: Generalized random processes and their extension to measures, (in Russian) Trudy Moskov. Obsc. 8 (1959), 497-518.

[7] Y. TAKAhashi: A note on Sazonov's Theorem, J. Fac. Sci. Hokkaido Univ. Ser. 1, Vol. 22, No. 3-4 (1972). 12̄6-131. 
[8] Y. UMEmURA: Measures on infinite dimensional vector spaces, Publ. Res. Inst. Mat. Sci. 1 (1965), 1-47.

[9] S. KOSHI and Y. TAKAHASHI: A Remark on quasi-invariant measure (to appear).

[10] L. Schwartz: Probabilités cylindriques et applications radonifiantes, J. Fac. Sci. Univ. Tokyo Sect. 1 A 18 (1971), 139-286.

(Received on July 1, 1974) 